\title{
Mesenteric Panniculitis in a Patient with Homozygous Factor $V$ Leiden Gene Mutation: A Case and Literature to Review
}

\begin{abstract}
Authors:
*Muhammad Sohaib Asghar, ${ }^{1}$ Abubakar Tauseef,' Durre Naman,, Maryam Zafar, ${ }^{1}$ Uzma Rasheed, ${ }^{2}$ Narmin Khan, ${ }^{1}$ Haris Alvi, ${ }^{1}$ Nimra Shaikh²

1. Dow University Hospital, Dow University of Health Sciences, Karachi, Pakistan

2. Liaquat National Hospital and Medical College, Karachi, Pakistan

*Correspondence to sohaib_asghar123@yahoo.com

Disclosure: $\quad$ The authors have declared no conflicts of interest.

Acknowledgements: Ethical approval was received from the institutional review board and consent to participate has been taken from the patient's guardian with informed verbal consent.

Received: $\quad 30.01 .20$

Accepted: $\quad 21.04 .20$

Keywords: Inflammation, mesenteric panniculitis, mesentery, factor $V$ Leiden, fibrosis, venous thrombosis.

Citation: $\quad$ EMJ. 2020;5[2]:108-112.

Abstract

A 30-year-old Asian male with a significant history of deep vein thrombosis and family history positive for pulmonary embolism presented with complaints of fever, nonradiating epigastric pain, and a sense of abdominal fullness. After the initial workup, ultrasonography of the whole abdomen was carried out which showed thrombus formation in the portal vein. A CT scan of the abdomen was performed, which showed findings suggestive of mesenteric panniculitis. Keeping the significant family history and imaging findings in mind, the clotting and thrombin profiles were analysed and came back positive for the factor $V$ Leiden gene (homozygous). A CT angiogram was performed to demonstrate extensive thrombosis throughout the abdominal vasculature with cavernous transformation. It is asserted that the chronic thrombosis on a background of factor $V$ mutation led towards chronic inflammation of the mesentery. To the authors' knowledge it is the first reported case of mesenteric panniculitis in a patient with factor $V$ homozygous gene mutation.
\end{abstract}

\section{INTRODUCTION}

Mesenteric panniculitis was first introduced by Jura in 1924, ', who termed it retractile mesenteritis. Since then, it has also been called sclerosing mesenteritis, mesenteric fibrosis, mesenteric lipodystrophy, and mesenteric panniculitis. ${ }^{2}$ It is a rare disease characterised by nonspecific chronic inflammation of the fatty tissue around the bowel mesentery. Its global prevalence is reported

to be $<1 \%{ }^{2}$ The clinical features of mesenteric panniculitis are diverse, with abdominal pain being the most common symptom. A frequent clinical sign of mesenteric panniculitis is the presence of a palpable abdominal mass. Abdominal CT scan with intravenous contrast is the best imaging modality for the diagnosis of mesenteric panniculitis. There was no consensus established about the mainstay of treatment in a review of the literature. 


\section{CASE PRESENTATION}

A 30-year-old Asian male with a significant history of deep vein thrombosis and family history of pulmonary embolism, presented with complaints of fever, nonradiating epigastric pain, nausea, vomiting, diarrhoea, and sense of fullness whenever he ate. The patient's symptoms included loss of appetite and fatigue associated with generalised weakness. He denied constipation, weight loss, bloating, a lump in the epigastric region, shortness of breath on lying down or during the night, yellowish discolouration of sclera and skin, pruritus, dark urine, and pale or bloody stools. He was started on low molecular weight heparin, intravenous normal saline, intravenous ciprofloxacin, antiemetic, and antipyretic for diarrhoea, vomiting, and fever.

The physical examination was unremarkable except for moderate pallor, distended abdomen, and severe tenderness in the epigastric region. The laboratory findings were haemoglobin ( $9.50 \mathrm{~g} / \mathrm{dL})$, mean corpuscular volume (71 fL), total leucocyte count (12,000 white blood cells/ $\mu \mathrm{L}$ with predominant neutrophilia), serum total bilirubin $(0.97 \mu \mathrm{mol} / \mathrm{L}$ with direct bilirubin of 0.56 $\mu \mathrm{mol} / \mathrm{L}$ ), C-reactive protein (299.4 $\mathrm{mg} / \mathrm{L}$ ), and erythrocyte sedimentation rate $(61 \mathrm{~mm} /$ hour). The rest of the laboratory findings were within normal limits including amylase, lipase, and viral markers (Epstein-Barr virus, cytomegalovirus, and hepatitis B and C). Autoimmune workup included antinuclear antibody and antiphospholipid antibody profiles which came back negative. Ultrasonography of the whole abdomen was done which showed thrombus formation in the portal vein. A CT scan of the abdomen with contrast was performed and showed generalised thickening of the mesentery with soft tissue infiltrates encircling the mesenteric vessels and mesenteric adenopathy, suggestive of mesenteric panniculitis (Figure 1). The differential considerations included acute pancreatitis, subacute intestinal obstruction, mesenteric panniculitis, chronic mesenteric ischaemia, and abdominal malignancy. Considering the ultrasonography findings, a CT angiogram was planned which showed thrombosis in the portal vein and its branches, splenic vein, and mesenteric veins with the cavernous transformation (Figure 2). Considering the significant family history and findings on the radiological imaging, clotting and thrombin profiles were analysed, which were found to be positive for the factor $V$ Leiden gene (homozygous), while the rest of the clotting profile including protein $C$, protein $\mathrm{S}$, antithrombin III, and homocysteine levels was within normal range.

The patient's clinical picture was suggestive of mesenteric panniculitis on a background of homozygous factor $V$ Leiden gene mutation. For further evaluation a laparoscopic biopsy was performed, which showed mixed inflammatory infiltrates with fat necrosis, confirming the diagnosis of mesenteric panniculitis. The patient was initially started on corticosteroids (prednisone: $1.0 \mathrm{mg} / \mathrm{kg} /$ day) for 3 weeks in addition to the empiric treatment, and anticoagulation was started with warfarin ( $7.5 \mathrm{mg} /$ day). His condition remained stable and the patient was discharged and followed-up in the ambulatory clinic every 3 months with symptomatic treatment. His steroids were tapered on follow-up (prednisone: $0.5 \mathrm{mg} /$ $\mathrm{kg} /$ day) with further tapering and eventually cessation on the resolution of symptoms after 6 months. No additional immunosuppressives were used in the management and a follow-up scan was recommended but could not be done. The patient was continued on anticoagulation with warfarin (5 mg/day).

\section{DISCUSSION}

Mesenteric panniculitis was first described in the literature by Jura in $1924^{1}$ as a rare entity of nonspecific chronic inflammation of the fatty bowel mesentery, with a global prevalence of $<1 \%$. In his study evaluating 7,620 abdominal CT scans, Daskalogiannaki et al. ${ }^{3}$ concluded mesenteric panniculitis has a prevalence of $0.6 \%$. The cause of the disease is still unknown but it has been hypothesised that autoimmune, ischaemic, and neoplastic factors play a role in the onset of this disease. ${ }^{3,4}$ Abdominal surgery and trauma are also potential causes. ${ }^{5,6}$ Infections such as malaria, tuberculosis, influenza, and syphilis can also act as a trigger in the onset of mesenteric panniculitis. The condition was previously treated as a separate clinical entity, ${ }^{7}$ but after a review of multiple case series Emory et al. $^{5}$ concluded that mesenteric panniculitis is one of the histological deviations of one disease process. 


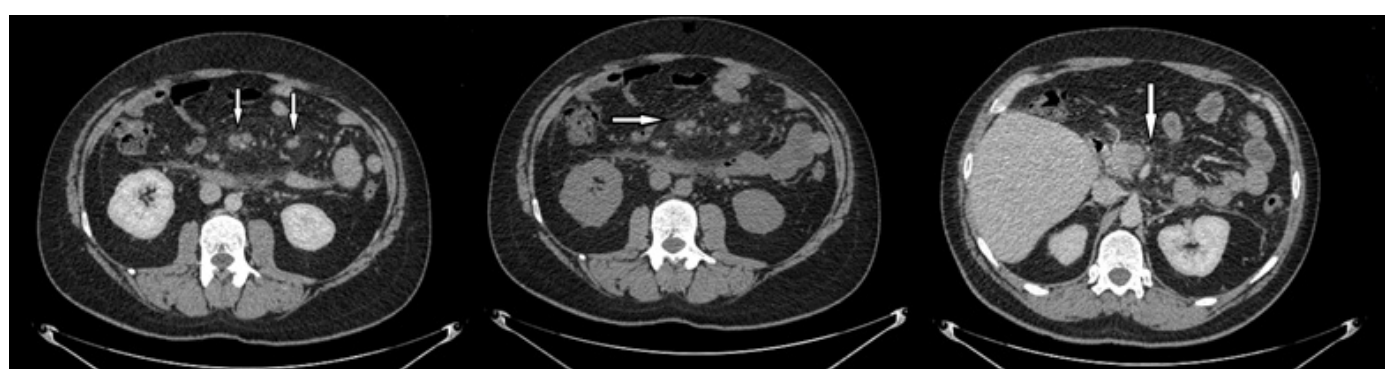

Figure 1: Abdominal CT scan.

CT of the abdomen displays increased attenuation and fat stranding, along with mesenteric lymph nodes with generalised thickening and soft tissue infiltrates within the mesentery.

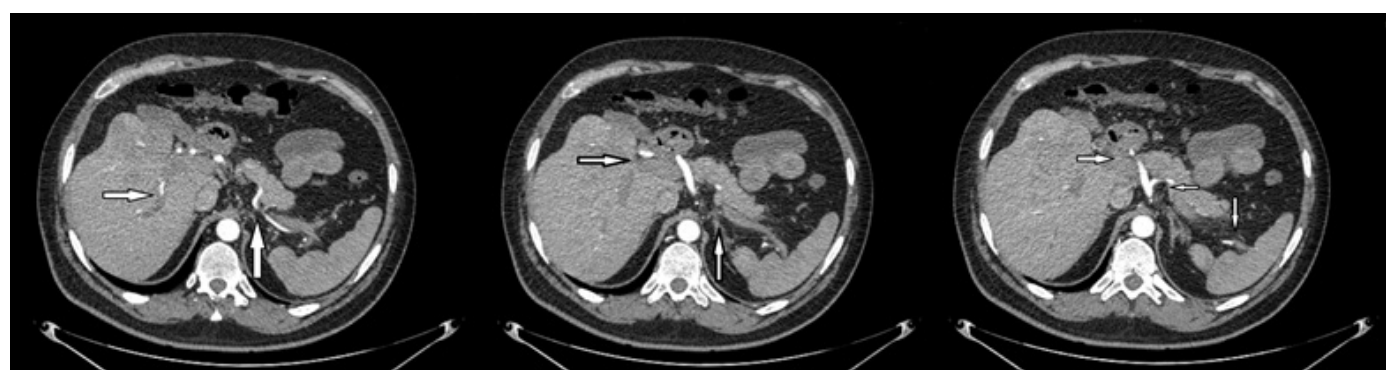

Figure 2: CT angiogram showing thrombosis in the portal, splenic, and mesenteric veins.

Sclerosing mesenteritis as a term is used for all variants but there is an overlap of clinical and pathological features resulting in many terminologies based on the predominant presenting pattern. It is classified as mesenteric panniculitis if there is marked chronic inflammation, mesenteric lipodystrophy in marked fatty degeneration and necrosis, and retractile mesenteritis if there is increased fibrosis.3,8 Mesenteric panniculitis can occur independently as well as in association with other diseases like Riedel's thyroiditis, retroperitoneal fibrosis, sclerosing cholangitis, granulomatous disease, vasculitis, rheumatic disease, malignancies, and pancreatitis. ${ }^{3,9}$ Sharma et al. ${ }^{8}$ describe the disease as affecting all age groups but typically manifestation is in the $6^{\text {th }}$ and $7^{\text {th }}$ decade of life, with a slight male predominance, though female predominance has also been reported in some studies. $^{3}$ The clinical features of mesenteric panniculitis are diverse, with abdominal pain being the most common symptom.4,7,10,11 Other symptoms include nausea, vomiting, anorexia, fever, weight loss, diarrhoea, or constipation. 3,4,7,10,12 Mesenteric panniculitis can also present completely asymptomatically $y^{3,4}$ and in one study, which evaluated 53 patients, the majority showed no symptoms. ${ }^{13}$ Mesenteric panniculitis can also present as protein-losing enteropathy. ${ }^{14}$ The most common clinical sign of mesenteric panniculitis is the presence of a palpable abdominal mass. ${ }^{7-}$ 10,12 Abdominal mass can result in incomplete bowel obstruction, ischaemia, and chylous ascites. ${ }^{12}$ Other signs, upon physical examination, can include abdominal tenderness, abdominal distention, and ascites. Laboratory findings are generally nonspecific.,10,13 Sharma et al., ${ }^{7}$ in their review article of 430 reference articles, described elevated erythrocyte sedimentation rate and C-reactive protein as the most common laboratory finding present in $88.0 \%$ and $86.5 \%$ of the patients, respectively. Another $17.2 \%$ had leucocytosis, $16.1 \%$ had anaemia, and $4.3 \%$ had low platelet levels. Plain radiographs are rarely helpful, barium studies might show the constriction of adjacent bowel or bowel loops distortion, ${ }^{15}$ but an abdominal CT scan is considered the best imaging modality for the diagnosis of mesenteric panniculitis. ${ }^{4}$ Mesenteric panniculitis is usually an incidental finding on $\mathrm{CT}$ scans following other 
unrelated conditions. In a study conducted in $2000,92 \%$ of the patients were diagnosed with mesenteric panniculitis without having any signs and symptoms. ${ }^{3}$ As identified in various works of literature, mesenteric panniculitis CT scans frequently contain the presence of a region of hyperattenuating mesenteric fat surrounding the mesenteric vessels (also known as misty mesentery), the presence of a soft tissue mass in the root of the mesentery, subcentimetre lymph nodes within the mesenteric fat, and preservation of the fat surrounding the mesenteric vessels forming a hypodense fat halo (also known as the fat ring sign). ${ }^{4,9,10,2,16}$ The most common CT scan finding is a soft tissue mass in the mesentery. ${ }^{9}$ The fat ring sign on the $\mathrm{CT}$ scan distinguishes mesenteric panniculitis from other mesenteric conditions such as carcinoid tumours, lymphoma, and carcinomatosis. ${ }^{9}$ Differential diagnoses sometimes cannot be narrowed down on imaging and histopathology alone, and biopsy is then required to confirm the diagnosis. ${ }^{12,15}$ Microscopically it presents as a variable mixture of chronic inflammation, fatty infiltration, and fibrosis., ${ }^{9,10}$ Prominent fibrosis with scant inflammation and fat necrosis is the most common histological manifestation..$^{10} \mathrm{~A}$ similar case has also been reported with the heterozygous state of factor $V$ Leiden, ${ }^{17}$ but the case described here presented with homozygous factor $V$ Leiden. According to different pieces of literature, various medications have been used to treat mesenteric panniculitis but still no consensus has been established about the mainstay of treatment.8,12 Medications that have been used to provide symptomatic relief include corticosteroids, ${ }^{7-10,12}$ tamoxifen, ${ }^{10}$ colchicine, ${ }^{9,18}$ cyclophosphamide, ${ }^{19}$ orally administered progesterone, ${ }^{9}$ and azathioprine, ${ }^{20}$ among others. A surgical approach is limited to mass biopsy or mass resection. ${ }^{12}$

\section{CONCLUSION}

This report highlights a case of a 30-year-old male diagnosed with mesenteric panniculitis in association with factor $V$ Leiden gene mutation. Although mesenteric panniculitis can occur with various other diseases, 3,9 its occurrence in a patient with homozygous factor $V$ Leiden gene mutation on a background of chronic thrombosis has yet to be reported, to the authors' knowledge. Furthermore, mesenteric vein thrombosis alone is also an unusual presentation for factor $V$ Leiden. The patient's clinical presentation was not favouring mesenteric ischaemia and neither of the abdominal imaging showed any evidence of ischaemia in the mesentery. Hence, it was assumed that the chronic nature of thrombosis might be a confounding factor in the diagnosis of mesenteric panniculitis. The patient was started on anticoagulants simultaneously, but the symptoms only improved after starting steroids, which were tapered and eventually stopped. The hypercoagulable workup demonstrated factor $V$ Leiden. No other plausible mechanism of mesenteric panniculitis, without any history of trauma, abdominal surgery, infection, or autoimmune or neoplastic pathogenesis, was considered. It is inferred that mesenteric panniculitis could be a nonspecific finding found incidentally on a CT scan, and is secondary to thrombosis which in turn is a result of factor $V$ Leiden mutation.

\section{References}

1. Jura V. [Retrattile -Caso clinico: Risultati sperimentali rilievi patogenetici considerazioni cliniche Policlinico (Sez Chir). 1927;34:535-59. (In Italian).

2. Khachaturian T, Hughes J. Mesenteric panniculitis. West J Med. 1988;148(6):700-1.

3. Daskalogiannaki M et al. CT evaluation of mesenteric panniculitis: prevalence and associated diseases. AJR Am J Roentgenol. 2000;174(2):427-31.
4. Badet $\mathrm{N}$ et al. Mesenteric panniculitis: still an ambiguous condition. Diagn Interv Imaging. 2015;96(3):251-7.

5. Emory TS et al. Sclerosing mesenteritis, mesenteric panniculitis and mesenteric lipodystrophy: a single entity. Am J Surg Pathol. 1997;21(4):392-8.

6. Jura V. [Sulla Mesenterite retrattile e sclerosante. Policlinico]. (Sez. Prat). 1924;31:575. (In Italian).

7. Monahan DW et al. Mesenteric panniculitis. South Med J.
1989;82(6):782-4.

8. Sharma $P$ et al. Sclerosing mesenteritis: a systematic review of 192 cases. Clin J Gastroenterol. 2017;10(2):103-11.

9. Horton $\mathrm{KM}$ et al. CT findings in sclerosing mesenteritis (panniculitis): spectrum of disease. Radiographics. 2003;23(6):1561-7.

10. Akram $\mathrm{S}$ et al. Sclerosing mesenteritis: clinical features, treatment, and outcome in ninety-two patients. Clin Gastroenterol Hepatol. 
2007;5(5):589-96.

11. Mindelzun RE et al. The misty mesentery on CT: differential diagnosis. AJR Am J Roentgenol. 1996;167(1):61-5.

12. Daumas A et al. Spontaneous resolution of severe, symptomatic mesocolic panniculitis: a case report. BMC Gastroenterol. 2012;12:59.

13. Kipfer RE et al. Mesenteric lipodystrophy. Ann Intern Med. 1974;80(5):582-8.

14. Rispo $A$ et al. Protein-loosing enteropathy in sclerosing mesenteritis. Eur Rev Med Pharmacol Sci. 2015;19(3):477-80.

15. McCrystal DJ et al. Mesenteric panniculitis: a mimic of malignancy. Aust N Z J Surg. 1998;68(3):237-9

16. van Putte-Katier $\mathrm{N}$ et al.

Mesenteric panniculitis: prevalence, clinicoradiological presentation and 5-year follow-up. Br J Radiol. 2014;87(1044):2014045.

17. Rothlein LR et al. Sclerosing mesenteritis successfully treated with a TNF antagonist. BMJ Case Rep. 2010;2010:bcr0720103145.

18. Iwanicki-Caron I et al. Successful management of symptoms of steroid-dependent mesenteric panniculitis with colchicine. Dig Dis Sci. 2006;51(7):1245-9

19. Bush RW et al. Sclerosing mesenteritis, response to cyclophosphamide. Arch Intern Med. 1986;146(3):503-5.

20. Bala A et al. Treatment of sclerosing mesenteritis with corticosteroids and azathioprine. Can J Gastrenterol. 2001;15(8):533-5. 